\title{
Simultaneous baldness and cosmic baldness and Kottler spacetime
}

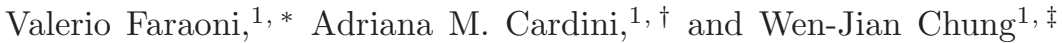 \\ ${ }^{1}$ Department of Physics and Astronomy, Bishop's University, \\ 2600 College Street, Sherbrooke, Québec, Canada J1M 1Z7
}

\begin{abstract}
The uniqueness of the Kottler/Schwarzschild-de Sitter solution of the vacuum Einstein equations with positive cosmological constant is discussed and certain putative alternatives are shown to either solve different equations or to be the KSdS solution in disguise. A simultaneous no-hair and cosmic no-hair theorem for the KSdS geometry in the presence of an imperfect fluid is proved.
\end{abstract}

\section{INTRODUCTION}

The Jebsen-Birkhoff theorem [1, 2] stating that the Schwarzschild geometry is the unique vacuum, spherically symmetric, and asymptotically flat solution of the Einstein equations is standard textbook material (see [3] for a review). Almost-Birkhoff theorems studying small deviations from spherical symmetry or vacuum have also been discussed [4, 5]. Relaxing the assumptions of the Jebsen-Birkhoff theorem to allow for an infinite distribution of matter leads to a variety of inhomogeneous universes [6, 7], which shows that there is no unique spherical solution with Friedmann-Lemaître-Robertson-Walker (FLRW) asymptotics. However, it is straightforward to extend the proof of the Jebsen-Birkhoff theorem to vacuum with a cosmological constant $\Lambda$ to deduce that the unique spherical solution of the vacuum Einstein equations in this case is the Kottler/Schwarzschild-de Sitter metric [8] (hereafter KSdS) if $\Lambda>0$ and the asymptotics are de Sitter, or the Schwarzschild-anti-de Sitter metric (SAdS) if $\Lambda<0$ and the asymptotics are antide Sitter. In locally static Schwarzschild-like coordinates $(T, R, \theta, \varphi)$ the KSdS metric has the form

$$
\begin{aligned}
d s^{2}= & -\left(1-\frac{2 m}{R}-H^{2} R^{2}\right) d T^{2}+\frac{d R^{2}}{1-\frac{2 m}{R}-H^{2} R^{2}} \\
& +R^{2} d \Omega_{(2)}^{2} .
\end{aligned}
$$

Here $m$ and $H=\sqrt{\Lambda / 3}$ are positive constants and $d \Omega_{(2)}^{2}=d \theta^{2}+\sin ^{2} \theta d \varphi^{2}$ is the line element on the unit 2 -sphere. The KSdS geometry plays the role of the prototypical black hole embedded in de Sitter space. The latter is extremely important for early universe inflation [9, 10] and is the late-time attractor of many dark energy and modified gravity models attempting to explain the current acceleration of the cosmic expansion [11] discovered in 1998 with type Ia supernovae. Likewise, anti-de Sitter space plays a prominent role in string theories and in the AdS/CFT correspondence [12] which have been the subject of a large literature (see [13] for recent reviews). It is surprising, therefore, that modern relativity

\footnotetext{
* vfaraoni@ubishops.ca

$\dagger$ acardini15@ubishops.ca

$\ddagger$ wchung13@ubishops.ca
}

textbooks do not mention the Jebsen-Birkhoff theorem in the presence of a cosmological constant, although occasionally one finds in the literature an explicit statement about the uniqueness of the Schwarzschild-(anti-)de Sitter space (e.g., [5, 14, 15]). A proof of the Jebsen-Birkhoff theorem extended to include a non-vanishing $\Lambda$ is available in Synge's 1960 textbook1] on general relativity [16]. More mathematically sophisticated proofs of the uniqueness of the KSdS and SAdS space are contained in old and recent references [17]. Similar to the situation of the Schwarzschild solution, uniqueness implies that the KSdS and SAdS solutions are stable with respect to perturbations, the stability being established in Refs. [18-21]. In spite of all this evidence, various works purport the existence of spherical solutions of the vacuum Einstein equations with $\Lambda>0$ which are alternatives to the KSdS one. This clearly cannot be true, or else these solutions must reduce to KSdS in disguise. There are also more general solutions of the Einstein equations representing central inhomogeneities embedded in FLRW spaces, which seem to reduce to alternatives to the KSdS solution in the special case when the FLRW "background" reduces to de Sitter. Again, this cannot be the case. Although these other authors presenting these solutions do not claim that they are alternatives to KSdS, nevertheless a situation was created which is unclear about the unique status of KSdS. To make things worse, enter alternative gravity. There is much interest in theories of gravity alternative to general relativity and in the study of their spherical solutions for various reasons. Although the JebsenBirkhoff theorem breaks down already in simple scalartensor gravity, some no-hair theorems persist and their relation with a positive cosmological constant has been discussed in the literature [22 25]. In particular, there are claims that spherical polytropic stars cannot match the KSdS exterior in scalar-tensor and $f(R)$ gravity 26, although the situation is still unclear in this regard [27]. Perhaps this happens because the KSdS solution is not adequate to describe inhomogeneous universes in these theories, but then one does not know which solution of the relevant field equations should be matched with the

\footnotetext{
${ }^{1}$ Synge does not mention Kottler's paper [8], nor does he refer to the KSdS solution as Schwarzschild-de Sitter but states that the metric is properly called "Schwarzschild solution" only when $\Lambda=0$.
} 
interior of a polytropic star, or with any local spherical object. It does not help these investigations if the situation is already confused in general relativity. Our purpose here is to make clarity about the status of KSdS space in general relativity and to reveal putative alternatives as KSdS in disguise due to the use of non-standard coordinate systems, or to identify them as genuinely different solutions which obey different field equations with matter sources. We then present a new no-hair/cosmic no-hair theorem related to KSdS space in the presence of an imperfect fluid. We use units in which Newton's constant $G$ and the speed of light $c$ are unity and we follow the notation of Ref. [28].

\section{UNIQUENESS OF THE KSDS METRIC}

The most general spherically symmetric line element in four spacetime dimensions can be written in the form

$$
d s^{2}=-A^{2}(t, R) d t^{2}+B^{2}(t, R) d R^{2}+R^{2} d \Omega_{(2)}^{2} .
$$

The vacuum Einstein equations

$$
G_{a b}=-\Lambda g_{a b}
$$

then yield

$$
\begin{aligned}
& \frac{2 \dot{B}}{R B}=0 \\
& \frac{2 B^{\prime}}{B^{3} R}-\frac{1}{B^{2} R^{2}}+\frac{1}{R^{2}}=\Lambda \\
& \frac{2 A^{\prime}}{A R}-\frac{B^{2}}{R^{2}}+\frac{1}{R^{2}}=-\Lambda B^{2} \\
& \frac{A^{\prime} B}{A}-B^{\prime}-\frac{R B^{2} \ddot{B}}{A^{2}}+\frac{R \dot{A} \dot{B} B^{2}}{A^{3}}-\frac{R A^{\prime} B^{\prime}}{A}+\frac{R A^{\prime \prime} B}{A} \\
& =-\Lambda R B^{3},
\end{aligned}
$$

where an overdot and a prime denote differentiation with respect to $t$ and $R$, respectively (the $(3,3)$ Einstein equation gives the same information as the $(2,2)$ equation). Using the consequence of Eq. (2.3) that $B=B(R)$, we drop the terms containing $\dot{B}$ or $\ddot{B}$ from Eq. (2.6). Equation (2.4) gives

$$
\left(\frac{R}{B^{2}}\right)^{\prime}=1-\Lambda R^{2}
$$

which is integrated to

$$
B^{2}(R)=\frac{1}{1+\frac{C}{R}-\frac{\Lambda R^{2}}{3}},
$$

where $C$ is an integration constant. By imposing that one recovers the Schwarzschild solution for a mass $m$ as $\Lambda \rightarrow 0$, one obtains $C=-2 m$ and

$$
B^{2}=\frac{1}{1-\frac{2 m}{R}-\frac{\Lambda R^{2}}{3}} .
$$

Equation (2.5) now gives

$$
\frac{2 A^{\prime}}{A}+\frac{1}{R}+\frac{\Lambda R^{2}-1}{R\left(1-\frac{2 m}{R}-\frac{\Lambda R^{2}}{3}\right)}=0,
$$

which can be written as

$$
\left(\ln A^{2}\right)^{\prime}=\left[\ln \left(1-\frac{2 m}{R}-\frac{\Lambda R^{2}}{3}\right)\right]^{\prime},
$$

and integrates to

$$
A^{2}(R)=\mathrm{e}^{D(t)}\left(1-\frac{2 m}{R}-\frac{\Lambda R^{2}}{3}\right)
$$

where $D(t)$ is an integration function of time. At this stage, one is not entitled to assume that $\dot{A}=0$. However, by rescaling the time coordinate according to

$$
d T=\mathrm{e}^{D(t) / 2} d t
$$

the spherically symmetric line element necessarily takes the static form

$$
\begin{aligned}
d s^{2}= & -\left(1-\frac{2 m}{R}-\frac{\Lambda R^{2}}{3}\right) d T^{2}+\frac{d R^{2}}{1-\frac{2 m}{R}-\frac{\Lambda R^{2}}{3}} \\
& +R^{2} d \Omega_{(2)}^{2} .
\end{aligned}
$$

This is the KSdS solution of the Einstein equations if $\Lambda>0$ (and then $H=\sqrt{\Lambda / 3}$ ), the SAdS solution if $\Lambda<0$, and it reduces to the Schwarzschild solution if $\Lambda=0$. The analysis of the spherical vacuum Einstein equations mirrors that, performed for $\Lambda=0$, which leads to the Jebsen-Birkhoff theorem in most relativity textbooks. If is therefore appropriate to speak of a generalized Jebsen-Birkhoff theorem when $\Lambda \neq 0$ and the KSdS solution is the unique solution of the vacuum Einstein equations with positive cosmological constant in spherical symmetry.

\section{PUTATIVE ALTERNATIVES TO KSDS}

Let us turn now to examining spherically symmetric solutions of the vacuum Einstein equations with $\Lambda>0$ which have been proposed as alternatives to the KSdS one, and to metrics which apparently contain alternatives to KSdS as special cases. Some ambiguity has been generated by the fact that these geometries have been presented in various coordinate systems, and different foliations of the KSdS spacetime can emphasize very different features (e.g., [29]).

\section{A. Abbassi-Meissner proposal}

Abbassi [30] and, ten years later, Meissner [31] reported the following metric as a new alternative to the KSdS geometry (here we adopt the notation of [31]):

$$
d s^{2}=-f(t, r) d t^{2}+\frac{\mathrm{e}^{2 H t}}{f(t, r)} d r^{2}+\mathrm{e}^{2 H t} r^{2} d \Omega_{(2)}^{2}
$$


where

$$
f(t, r)=h(t, r)+\sqrt{h^{2}(t, r)+H^{2} r^{2} \mathrm{e}^{2 H t}},
$$

and

$$
h(t, r)=\frac{1}{2}\left(1-H^{2} r^{2} \mathrm{e}^{2 H t}-\frac{2 m}{r} \mathrm{e}^{-H t}\right),
$$

$m$ is a constant mass parameter and $H$ is the Hubble constant of the de Sitter background given by $H^{2}=\Lambda / 3$. The areal radius of this spherically symmetric geometry is $R(t, r)=a(t) r=\mathrm{e}^{H t} r$. Making use of the relation between differentials $d r=a^{-1}(d R-H R d t)$, one rewrites the line element 3.1 in terms of the areal radius as

$$
\begin{aligned}
d s^{2}= & -2 h(0, R) d t^{2}-\frac{2 H R}{f(0, R)} d t d R+\frac{d R^{2}}{f(0, R)}+R^{2} d \Omega_{(2)}^{2} \\
= & -\left(1-\frac{2 m}{R}-H^{2} R^{2}\right) d t^{2} \\
& -\frac{4 H R}{1-\frac{2 m}{R}-H^{2} R^{2}+\sqrt{\left(1-\frac{2 m}{R}-H^{2} R^{2}\right)^{2}+4 H^{2} R^{2}}} d t d R \\
& +\frac{2 d R^{2}}{1-\frac{2 m}{R}-H^{2} R^{2}+\sqrt{\left(1-\frac{2 m}{R}-H^{2} R^{2}\right)^{2}+4 H^{2} R^{2}}}+R^{2} d \Omega_{(2)}^{2} .
\end{aligned}
$$

By introducing a new time coordinate $T$ defined by

$$
d T=d t+\beta(t, R) d R,
$$

with $\beta(t, R)$ a function to be determined, and

$$
A_{0}(R) \equiv 1-\frac{2 m}{R}-H^{2} R^{2}=2 h(0, R),
$$

one obtains

$$
\begin{aligned}
d s^{2}= & -A_{0} d T^{2}+\left(-A_{0} \beta^{2}+\frac{4 H R \beta}{A_{0}+\sqrt{A_{0}^{2}+4 H^{2} R^{2}}}\right. \\
& \left.+\frac{2}{A_{0}+\sqrt{A_{0}^{2}+4 H^{2} R^{2}}}\right) d R^{2}+R^{2} d \Omega_{(2)}^{2} \\
& +2\left(\beta A_{0}-\frac{2 H R}{A_{0}+\sqrt{A_{0}^{2}+4 H^{2} R^{2}}}\right) d T d R .
\end{aligned}
$$

By setting

$$
\beta(R)=\frac{2 H R}{A_{0}\left(A_{0}+\sqrt{A_{0}^{2}+4 H^{2} R^{2}}\right)}
$$

the cross-term in $d T d R$ is eliminated and the line element assumes the diagonal and locally static form

$$
\begin{aligned}
d s^{2}= & -A_{0}(R) d T^{2}+\frac{2}{A_{0}(R)+\sqrt{A_{0}^{2}(R)+4 H^{2} R^{2}}} \\
& \cdot\left[1+\frac{2 H^{2} R^{2}}{A_{0}\left(A_{0}+\sqrt{A_{0}^{2}+4 H^{2} R^{2}}\right)}\right] d R^{2}+R^{2} d \Omega_{(2)}^{2},
\end{aligned}
$$

which is not of the KSdS form. A posteriori one can check that $d T=d t+\beta d R$ is an exact differential (i.e., the time coordinate $T$ is well defined) by noting that it is closed,

$$
\frac{\partial(1)}{\partial R}=0=\frac{\partial \beta}{\partial t}
$$

Although claiming a new solution alternative to the KSdS one, Abbassi [30] mentions a coordinate transformation that brings the line element (3.1) to the standard KSdS form, but this coordinate change fails to do so. Moreover, this author ascribes different physical meanings to the same geometry described in different coordinate systems. The geometry, however, must be coordinate independent. In particular, the static character of the metric is shown by the existence of a timelike Killing vector field. In spite of what stated in 30, 31 the diagonal metric (3.1) does not solve the vacuum Einstein equations $R_{a b}=\Lambda g_{a b}$ but it is generated by matter sources. For example, there is a radial mass flow given by 


$$
\begin{aligned}
T_{01}= & \frac{1}{8 \pi} \frac{d\left(\ln B^{2}\right)}{d T}=\frac{H \dot{H} R^{2}}{2 \pi\left(A_{0}+\sqrt{A_{0}^{2}+4 H^{2} R^{2}}\right) \sqrt{A_{0}^{2}+4 H^{2} R^{2}}} \\
& \cdot\left\{-1+\frac{A_{0}\left(A_{0} \sqrt{A_{0}^{2}+4 H^{2} R^{2}}+A_{0}^{2}+2 H^{2} R^{2}\right)}{\sqrt{A_{0}^{2}+4 H^{2} R^{2}}\left[A_{0}\left(A_{0}+\sqrt{A_{0}^{2}+4 H^{2} R^{2}}\right)+2 H^{2} R^{2}\right]}\right\} .
\end{aligned}
$$

\section{B. McVittie and generalized McVittie solutions}

The McVittie solution was originally introduced to model the effect of the cosmological expansion on local systems 32 and has been the subject of much recent literature 33,36$]$. It represents a central inhomogeneity (possibly a black hole) embedded in a FLRW space. The source for the exterior McVittie metric is a fluid with energy density $\rho(t)$ which depends only on time, and pressure $P(t, r)$ which depends on both time and radius. The line element can be cast in the form 35, 37.

$$
\begin{aligned}
d s^{2}= & -\left[1-\frac{2 m}{R}-H^{2}(t) R^{2}\right] d t^{2}-\frac{2 H(t) R}{\sqrt{1-2 m / R}} d t d R \\
& +\frac{d R^{2}}{1-2 m / R}+R^{2} d \Omega_{(2)}^{2},
\end{aligned}
$$

where $m$ is a positive constant related to the mass of the central object and $H(t)$ is the Hubble parameter of the FLRW space in which this object is embedded. When the FLRW "background" reduces to de Sitter, $H=$ const., the transformation to the coordinate $T$ given by

$$
d T=d t+\frac{H R d R}{\sqrt{1-\frac{2 m}{R}}\left(1-\frac{2 m}{R}-H^{2} R^{2}\right)}
$$

reduces the metric to the KSdS form 1.10. Therefore, the McVittie metric with $H=$ const. is not an alternative to KSdS but it contains it as a special case.

In the literature there is also a class of "generalized McVittie solutions" in which, contrary to the original McVittie one, there is a spacelike radial heat flow $q^{\mu}=(0, q, 0,0)[33,38]$. McVittie spaces are also solutions of cuscuton theory (a special case of HořavaLifschitz gravity [39]) and generalized McVittie spaces are also solutions of Horndeski gravity and shape dynamics [40]. They are substantially more complicated than the McVittie one, but they also reduce to the KSdS geometry when the background is de Sitter [33, 38], in which case the spacelike radial energy flow $q^{a}$ vanishes.

\section{Non-rotating Thakurta solution}

The Thakurta solution of the Einstein equations [41] describes a rotating black hole embedded in a FLRW universe. When the angular momentum is set to zero and the cosmological background is chosen to be de Sitter, one obtains an apparent alternative to KSdS but this is not the case, as explained below. The non-rotating Thakurta solution was recently analyzed in detail in [42], see also [43, 44]. The line element is

$$
\begin{aligned}
d s^{2} & =a^{2}(\eta)\left[-\left(1-\frac{2 m}{r}\right) d \eta^{2}+\frac{d r^{2}}{1-2 m / r}+r^{2} d \Omega_{(2)}^{2}\right] \\
& =-\left(1-\frac{2 m}{r}\right) d t^{2}+\frac{a^{2} d r^{2}}{1-2 m / r}+a^{2} r^{2} d \Omega_{(2)}^{2},
\end{aligned}
$$

where $a(\eta)$ is the scale factor of the FLRW background, $\eta$ and $t$ are its conformal and comoving times, respectively, with $d t=a d \eta$, and $m$ is a constant mass parameter. The line element (3.15) is manifestly conformal to the Schwarzschild one. By using the areal radius $R(t, r)=a(t) r$ and the relation between differentials $d r=\frac{d R}{a}-H R d \eta$ (where $H \equiv \dot{a} / a$ and an overdot denotes differentiation with respect to the comoving time $t)$, the line element is rewritten as

$$
\begin{aligned}
d s^{2}= & -\left(1-\frac{2 M(t)}{R}-\frac{H^{2} R^{2}}{1-2 M(t) / R}\right) d t^{2} \\
& +\frac{d R^{2}}{1-2 M(t) / R}-\frac{2 H R}{1-2 M(t) / R} d t d R+R^{2} d \Omega_{(2)}^{2},
\end{aligned}
$$

where

$$
M(t) \equiv m a(t) .
$$

The cross-term in $d t d R$ can be eliminated from this line element [45]. We use $A(t, R) \equiv 1-2 M / R=1-2 m / r$ and a new time coordinate $T$ defined by

$$
d T=\frac{1}{F}\left(d t+\frac{H R}{A^{2}-H^{2} R^{2}} d R\right)
$$

where $F(t, R)$ is an integrating factor satisfying

$$
\frac{\partial}{\partial R}\left(\frac{1}{F}\right)=\frac{\partial}{\partial t}\left(\frac{H R}{F\left(A^{2}-H^{2} R^{2}\right)}\right)
$$

to guarantee that $d T$ is an exact differential. Straightforward manipulations bring the line element to the diagonal gauge

$$
\begin{aligned}
d s^{2}= & -\left(1-\frac{2 M}{R}-\frac{H^{2} R^{2}}{1-\frac{2 M}{R}}\right) F^{2} d T^{2} \\
& +\frac{d R^{2}}{1-\frac{2 M}{R}-\frac{H^{2} R^{2}}{1-2 M / R}}+R^{2} d \Omega_{(2)}^{2} .
\end{aligned}
$$


Using the form (3.15) of the metric, the Einstein equations give [42]

$$
\begin{aligned}
& G_{0}{ }^{0}=8 \pi T_{0}{ }^{0}=-\frac{3 H^{2}}{A}, \\
& G_{1}{ }^{0}=8 \pi T_{1}{ }^{0}=-\frac{2 m H}{r^{2} A^{2}}, \\
& G_{1}{ }^{1}=8 \pi T_{1}{ }^{1}=8 \pi T_{2}{ }^{2}=8 \pi T_{3}{ }^{3}=-\frac{1}{A}\left(H^{2}+\frac{2 \ddot{a}}{a}\right) .
\end{aligned}
$$

Assume a de Sitter background with $H=\sqrt{\Lambda / 3}$ and $a(t)=a_{0} \mathrm{e}^{H t}$; then the time-radius Einstein equation (3.22) satisfied by the non-rotating Thakurta solution clearly cannot reduce to the corresponding equation satisfied by the KSdS metric, which would instead give $8 \pi T_{1}{ }^{0}=-\Lambda g_{1}{ }^{0}=0$ (the vanishing of $T_{1}{ }^{0}$ means that, because the cosmological constant is repulsive, it does not accrete onto a black hole and there is no radial energy flow). The two equations only coincide in the trivial cases when $m=0$ (de Sitter space) or when $a$ = const. (Minkowski background). These two equations cannot coincide because, as stated clearly in [42, 43], the source of the non-rotating Thakurta geometry is not a perfect fluid, to which the cosmological constant can be reduced, but is instead an imperfect one with a spacelike radial heat flow which has components $q_{u}=\left(0,-2 m \dot{a} a A^{-3 / 2} / r^{2}, 0,0\right)$ in coordinates $(t, r, \theta, \varphi)$ [42].

It has been shown in Refs. [36, 46] that the nonrotating Thakurta solution is the late time attractor of generalized McVittie solutions, but these references 2 did not recognize the geometry as a special case of the less known Thakurta solution and called it "comoving mass solution" instead. The non-rotating Thakurta solution is also the limit to general relativity of a class of solutions of Brans-Dicke theory found in Ref. [47] as the Brans-Dicke parameter $\omega \rightarrow \infty$ [36, 46].

\section{Castelo Ferreira metric}

Another line element which resembles, or even reduces to some of the previous ones for special parameter values was introduced by Castelo Ferreira [48]

$$
\begin{aligned}
d s^{2}= & -\left[1-\frac{2 m}{R}-H^{2} R^{2}\left(1-\frac{2 m}{R}\right)^{\alpha}\right] d t^{2}+\frac{d R^{2}}{1-\frac{2 m}{R}} \\
& -2 H R\left(1-\frac{2 m}{R}\right)^{\frac{\alpha-1}{2}} d t d R+R^{2} d \Omega_{(2)}^{2},
\end{aligned}
$$

where $\alpha$ and $m$ are constants and $H=H(t)$ is the Hubble parameter of the FLRW "background". This geometry does not satisfy the vacuum Einstein equations $G_{a b}=$ $-\Lambda g_{a b}$ but is sourced by an imperfect fluid which has different tangential and radial pressures if $\alpha \neq 0$ [48]. The metric 3.24 reduces to the McVittie metric in the form (3.13) when $\alpha=0$ (in which case the two pressures coincide). In spite of superficial similarities, it does not reduce to the non-rotating Thakurta solution (3.16) for $\alpha=-1$. Similarities and differences may be misleading because they depend on the coordinates adopted. Let us change the time coordinate $t \rightarrow T$, where $T$ is defined by

$$
d T=\frac{1}{F}(d t+\beta d R)
$$

where $1 / F$ is an integrating factor and $\beta(t, R)$ is a function to be determined. The line element (3.24) becomes

$$
\begin{aligned}
d s^{2}= & -\left[1-\frac{2 m}{R}-H^{2} R^{2}\left(1-\frac{2 m}{R}\right)^{\alpha}\right] F^{2} d T^{2} \\
& +\left\{-\left[1-\frac{2 m}{R}-H^{2} R^{2}\left(1-\frac{2 m}{R}\right)^{\alpha}\right] \beta^{2}+\frac{1}{1-\frac{2 m}{R}}+2 H R \beta\left(1-\frac{2 m}{R}\right)^{\frac{\alpha-1}{2}}\right\} d R^{2} \\
& +2 F\left\{\left[1-\frac{2 m}{R}-H^{2} R^{2}\left(1-\frac{2 m}{R}\right)^{\alpha}\right] \beta-H R\left(1-\frac{2 m}{R}\right)^{\frac{\alpha-1}{2}}\right\} d T d R+R^{2} d \Omega_{(2)}^{2} .
\end{aligned}
$$

By setting

$$
\beta(t, R)=\frac{H R\left(1-\frac{2 m}{R}\right)^{\frac{\alpha-1}{2}}}{1-\frac{2 m}{R}-H^{2} R^{2}\left(1-\frac{2 m}{R}\right)^{\alpha}}
$$

\footnotetext{
2 Ref. [43] studied the same geometry for different purposes and did not identify it with the Thakurta solution.
}

the cross-term in $d T d R$ is eliminated and one obtains the line element in the diagonal gauge

$$
\begin{aligned}
d s^{2}= & -\left[1-\frac{2 m}{R}-H^{2} R^{2}\left(1-\frac{2 m}{R}\right)^{\alpha}\right] F^{2} d T^{2} \\
& +\frac{d R^{2}}{1-\frac{2 m}{R}-H^{2} R^{2}\left(1-\frac{2 m}{R}\right)^{\alpha}}+R^{2} d \Omega_{(2)}^{2} .
\end{aligned}
$$


If the background is de Sitter then $H=$ const., $\beta=\beta(R)$, and $F=1$, and the line element (3.28) reduces to the non-rotating Thakurta solution (3.20) for $\alpha=-1$ and to the KSdS form (1.1) (which is a special case of McVittie) for $\alpha=0$. It is clear, however, that in the general case the geometry is different from the KSdS one.

\section{SIMULTANEOUS BALDNESS AND COSMIC BALDNESS}

Cosmic no hair theorems state that, with a few exceptions (Bianchi models which are overdense and collapse before the cosmological constant can come to dominate the dynamics), de Sitter space is an attractor in the late time dynamics of the universe [49]. Similarly, under reasonable conditions, no-hair theorems for black holes exclude the possibility of fields in the exterior spacetime of black holes which would make the geometry deviate from Schwarzschild 23]. Since the KSdS geometry brings together black hole physics and de Sitter cosmology, presumably simultaneous no-hair and cosmic no-hair results, pointing to the KSdS spacetime as the final attractor state, should be valid in the presence of a positive cosmological constant, spherical symmetry, and a central inhomogeneity. This idea is supported by the uniqueness of the KSdS solution in vacuo and by its perturbative stability [18 21]. In the following we derive a non-perturbative result in this direction which is motivated by the presence of imperfect fluids in the solutions of the Einstein equations discussed in the previous sections.

Consider the Einstein equations with matter

$$
G_{a b}=-\Lambda g_{a b}+8 \pi T_{a b}
$$

and assume spherical symmetry, in which case the line element is given by Eq. (2.1). Assume that the solution of the Einstein equations is asymptotically de Sitter, that is, that there is a de Sitter-like cosmological horizon of areal radius $R_{H}$ and the solution of the Einstein equations (4.1) reduces to (1.1) a $8 \rightarrow R_{H}^{-}$. The Einstein

\footnotetext{
3 The coordinates $(t, R, \theta, \varphi)$ are expected to break down when $R>R_{H}$ or when $R$ becomes smaller than the black hole horizon $R_{B H}$ that may be present. Outside of the region $R_{B H} \leq R \leq$ $R_{H}$, the geometry is not expected to be locally static, as in KSdS space.
}

equations are now

$$
\begin{aligned}
& \frac{\dot{B}}{B R}=4 \pi T_{01} \\
& A^{2}\left(\frac{2 B^{\prime}}{B^{3} R}-\frac{1}{B^{2} R^{2}}+\frac{1}{R^{2}}\right)=\Lambda A^{2}+8 \pi T_{00} \\
& \frac{2 A^{\prime}}{A R}-\frac{B^{2}}{R^{2}}+\frac{1}{R^{2}}=-\Lambda B^{2}+8 \pi T_{11} \\
& \frac{A^{\prime} B}{A}-B^{\prime}-\frac{R B^{2} \ddot{B}}{A^{2}}+\frac{R \dot{A} \dot{B} B^{2}}{A^{3}}-\frac{R A^{\prime} B^{\prime}}{A}+\frac{R A^{\prime \prime} B}{A} \\
& =\left(-\Lambda R^{2}+8 \pi T_{22}\right) \frac{B^{3}}{R} .
\end{aligned}
$$

Further assume that matter is described by an imperfect fluid with constant equation of state and a purely spatial radial heat flow (of the kind considered in the previous section),

$$
\begin{gathered}
T_{a b}=(P+\rho) u_{a} u_{b}+P g_{a b}+q_{a} u_{b}+q_{b} u_{a}, \\
P=w \rho, \quad w=\text { const. } \\
u^{a} u_{a}=-1, \quad q^{c} u_{c}=0 .
\end{gathered}
$$

The fluid 4-velocity and the radial energy flow have components

$$
\begin{aligned}
& u^{\mu}=\left(|A|^{-1}, 0,0,0\right), \quad u_{\mu}=(-|A|, 0,0,0), \\
& q^{\mu}=(0, q, 0,0), \quad q_{\mu}=\left(0, B^{2} q, 0,0\right) .
\end{aligned}
$$

The components of the stress-energy tensor (4.6) are

$$
\begin{aligned}
& T_{00}=A^{2} \rho, \\
& T_{01}=-|A| B^{2} q, \\
& T_{11}=B^{2} P, \\
& T_{22}=R^{2} P, \\
& T_{33}=R^{2} P \sin ^{2} \theta .
\end{aligned}
$$

$T_{01}>0$ and $q<0$ correspond to radial inflow, while $T_{01}<0$ and $q>0$ to outflow.

In the case of inflow $q<0$, Eq. (4.2) yields

$$
\left(B^{2}\right)^{\cdot}=-8 \pi|A| B^{4} R q>0,
$$

therefore the metric component $B^{2}=g_{11}$ increases with time. Assuming the metric coefficients to be continuous and differentiable, there are then two possibilities: either $B^{2}(t, R) \rightarrow+\infty$ for any fixed $R$ as $t \rightarrow+\infty$ (or as $t \rightarrow$ 
$t_{\max }$ if there is a singularity at a finite future $\left.t_{\max }\right)$, or $B^{2}(t, R)$ has an horizontal asymptote as $t \rightarrow+\infty$.

Let us consider the first case. The apparent horizons are located by the covariant equation $\nabla^{c} R \nabla_{c} R=0$, equivalent to $1 / B^{2}=0$ in the coordinates used. If $B^{2} \rightarrow+\infty$ as $t \rightarrow+\infty$ or as $t \rightarrow t_{\max }$, then at late times all points of space at any value of $R$ lie arbitrarily close to an apparent horizon. This situation is familiar in cosmology: it corresponds to a phantom universe in which there is a Big Rip singularity at a finite time $t_{\max }$ and the apparent horizon (which has areal radius $R_{A H}=H^{-1}$ in a spatially flat FLRW cosmos [36]) shrinks around a comoving observer because the expansion of the universe super-accelerates, i.e., $\dot{H}=-4 \pi(P+\rho)>0$ [51]. By contrast, in a de Sitter space the Hubble parameter $H$ remains constant although the expansion itself accelerates, $\ddot{a}>0$. In a universe dominated by non-phantom dark energy (other than the cosmological constant), it is instead $\dot{H}<0$ while $\ddot{a}>0$. These phantom asymptotics contradict our assumption of de Sitter asymptotics and, therefore, we discard this possibility.

There remains the case in which $B^{2}(t, R)$ asymptotes to a function $B_{0}^{2}(R)$ of $R$ as $t \rightarrow+\infty$. In this case $\dot{B} \rightarrow 0$ as $t \rightarrow+\infty$ (which also implies that the apparent horizons located by the equation $1 / B^{2}=0$ become less and less dynamical). Then Eq. (4.16) implies that the radial flow $q \rightarrow 0$ as $t \rightarrow+\infty$. In conjunction with Eq. (4.11), the differentiation of Eq. (4.3) yields

$$
8 \pi \dot{\rho}=\frac{2}{R}\left(\frac{B^{\prime}}{B^{3}}\right)^{\cdot}-\frac{1}{R^{2}}\left(\frac{1}{B^{2}}\right)^{\cdot} \rightarrow 0 \quad \text { as } t \rightarrow+\infty .
$$

The assumption that $P=w \rho$ with constant $w$ (or with $w=w(R))$ then implies that also $\dot{P} \rightarrow 0$ as $t \rightarrow+\infty$. Equations (4.13) and (4.4) give

$$
8 \pi \dot{P}=\frac{2}{R}\left(\frac{A^{\prime}}{A B^{2}}\right)^{\cdot}+\frac{1}{R^{2}}\left(\frac{1}{B^{2}}\right)^{\cdot} \approx \frac{2}{R B^{2}}\left(\frac{A^{\prime}}{A}\right)^{\cdot} \rightarrow 0
$$

as $t \rightarrow+\infty$. Therefore, also $A^{2}$ becomes timeindependent, and the metric becomes static as $t \rightarrow+\infty$.

To make progress, consider the covariant conservation equation $\nabla^{b} T_{a b}=0$ for the imperfect fluid stress-energy tensor (4.6), which yields

$$
\begin{aligned}
& u_{a} u^{b} \nabla_{b}(P+\rho)+\left[(P+\rho) u_{a}+q_{a}\right] \nabla^{b} u_{b} \\
& +\left[(P+\rho) u_{b}+q_{b}\right] \nabla^{b} u_{a}+\nabla_{a} P+u^{b} \nabla_{b} q_{a}+u_{a} \nabla^{b} q_{b}=0 .
\end{aligned}
$$

Projecting this equation onto the time direction $u^{a}$ of comoving observers and using the orthogonality of 4velocity and 4-acceleration $u^{a} \nabla_{b} u_{a}=0$, one obtains

$$
-\dot{\rho}-(P+\rho) \nabla^{b} u_{b}+u^{a} q^{b} \nabla_{b} u_{a}+u^{a} u^{b} \nabla_{b} q_{a}-\nabla^{b} q_{b}=0 \text {. }
$$

At late times $q^{c}$ and $\dot{\rho}$ disappear from this equation, as we have deduced above, leaving

$$
(P+\rho) \nabla^{b} u_{b} \simeq 0 .
$$

In general $\nabla^{b} u_{b}$ is different from zero (indeed, since the geometry must be asymptotically de Sitter at large radii, $\nabla^{b} u_{b}$ reduces to $3 H>0$ there) and we are left with $P+\rho \rightarrow 0$ as $t \rightarrow+\infty$. Either the matter fluid reduces to a cosmological constant, in which case the vacuum uniqueness theorem for $\mathrm{KSdS}$ holds, or else both $\rho$ and $P=w \rho$ become subdominant and the cosmological constant dominates the expansion at late times while $\rho$ and $P$ become unimportant. Also in this case the solution reduces to KSdS.

If instead there is outflow $q>0$, then

$$
\left(B^{2}\right)^{\cdot}=-8 \pi|A| B^{4} R q<0
$$

and, since $B^{2}$ is bounded from below by zero and it decreases as $t \rightarrow+\infty$, it must have a horizontal asymptote with $B^{2}(t, R) \rightarrow B_{0}^{2}(R)^{+}$for any fixed $R$ as $t \rightarrow+\infty$. Then $\dot{B} \rightarrow 0$ and $q \rightarrow 0$. The reasoning made in the case with inflow is then repeated from this point, reaching the same conclusion. Hence it is proved that, assuming spherical symmetry, $\Lambda>0$ and spatial de Sitter asymptotics, and an imperfect fluid with constant equation of state and purely spatial radial energy flow, the late time solution of the Einstein equations must be the KSdS geometry.

As a special case, one can consider a perfect fluid by setting $q^{a}=0$. In this case $T_{01}=0$ and Eq. (4.12) gives $B=B(R)$. It is then straightforward to prove that it must be $P=-\rho$ and that the KSdS geometry can be the only solution (this simple proof for a perfect fluid was already given in Ref. [33]).

\section{CONCLUSIONS}

As seen in Sec. II the KSdS solution is the unique spherically symmetric solution of the vacuum Einstein equations with positive cosmological constant. This result is a simple generalization of the ordinary JebsenBirkhoff theorem [1, 2], which makes the same assumptions except that it assumes $\Lambda=0$, and goes hand-inhand with the perturbative analyses which established the stability of the KSdS solution [18 21]. Putative alternative solutions of the Einstein equations under the same conditions either solve different equations (for example, including an imperfect fluid with spacelike radial flow) or are just the KSdS solution in disguise in an unusual coordinate system.

Since no-hair theorems reinforce the uniqueness of the Schwarzschild geometry [23] and cosmic no-hair theorems establish that the de Sitter space is the unique late-time attractor in cosmology (with few exceptions [49]), it is reasonable to expect that similar theorems should hold for the KSdS spacetime, which is usually interpreted as describing a Schwarzschild black hole embedded in de Sitter space. Such theorems would prove the uniqueness of the KSdS solution. We have proved a result of this kind by assuming spherical symmetry and the presence of an imperfect fluid with constant equation of state 
$P=w \rho$ and a purely spatial radial energy flow, which is a rather common ingredient in the construction of solutions of the Einstein equations representing spherical inhomogeneous universes (e.g., [33, 34, 38, 42, 50]). The theorem proved in Sec. IV does not contradict the previous statement of Sec. III that the non-rotating Thakurta solution is the late-time attractor of generalized McVittie solutions [46] because, in this case, the asymptotics are (time-dependent) FLRW and not de Sitter, which was one of the assumptions in our theorem. Simultane- ous cosmic no-hair/no-hair theorems more general than the one in Sec.IV (possibly including anisotropy) will be investigated in the future.

\section{ACKNOWLEDGMENTS}

We thank Jeremy Coté and Thomas Gobeil for discussions. This work is supported by the Natural Sciences and Engineering Research Council of Canada.
[1] J.T. Jebsen, Ark. Mat. Ast. Fys. (Stockholm) 15, nr. 18 (1921), reprinted in Gen. Relat. Gravit. 37, 2253 (2005).

[2] G.D. Birkhoff, Relativity and Modern Physics (Harvard University Press, Cambridge, USA 1923).

[3] P.T. Chruściel, J. Lopes Costa, and M. Heusler, Living Rev. Relativity 15, 7 (2012).

[4] R. Goswami and G.F.R. Ellis, Gen. Relat. Gravit. 43, 2157 (2011); Gen. Relat. Gravit. 44, 2037 (2012); G.F.R. Ellis and R. Goswami, Gen. Relat. Gravit. 45, 2123 (2013).

[5] A.M Nzioki, R. Goswami, and P.K.S. Dunsby, Phys. Rev. D 89, 064050 (2014).

[6] A. Krasiński, Inhomogeneous Cosmological Models (Cambridge University Press, Cambridge, UK, 1997).

[7] H. Stephani, D. Kramer, M. MacCallum, C. Hoenselaers, and E. Herlt, Exact Solutions of Einsteins Field Equations (Cambridge University Press, Cambridge, 2003).

[8] F. Kottler, Ann. Phys. (Leipzig) 56, 401 (1918).

[9] A.D. Linde, Particle Physics and Inflationary Cosmology (Harwood, Chur, Switzerland, 1990).

[10] A.R. Liddle and D.H. Lyth, Cosmological Inflation and Large-Scale Structure (Cambridge University Press, Cambridge, 2000).

[11] L. Amendola and S. Tsujikawa, Dark Energy, Theory and Observations (Cambridge University Press, Cambridge, UK, 2010).

[12] J.M. Maldacena. Int. J. Theor. Phys. 38, 1113 (1999).

[13] V.E. Hubeny, Class. Quantum Grav. 32, 124010 (2015); A.V. Ramallo, Introduction to the AdS/CFT Correspondence, in Springer Proc. Phys. 161, 411 (2015).

[14] H.-J. Schmidt, Gen. Relat. Gravit. 45, 395 (2013).

[15] A.C. Fabian and A.N. Lasenby, in General Relativity: The Most Beautiful of Theories, ed. by C. Rovelli, De Gruyter Studies in Mathematical Physics vol. 28 (De Gruyter, Berlin, 2015).

[16] J.L. Synge, Relativity: The General Theory (North Holland, Amsterdam, 1960).

[17] M. Boucher, G.W. Gibbons, and G.T. Horowitz Phys. Rev. D 30, 2447 (1984); H. Kodama, J. Korean Phys. Soc. 45 S68 (2004); P.G. LeFloch and L. Rozoy, Compt. Rendu. Acad. Sci. Paris Ser. I 348, 1129 (2010); A.K.M. Masood-ul-Alam and W. Yu, Comm. Analys. Geom. 23, 377 (2015).

[18] J. Guven and D. Nuñez, Phys. Rev. D 42, 2577 (1990).

[19] R. Balbinot and E. Poisson, Phys. Rev. D 41, 395 (1990).

[20] F. Mellor and I. Moss, Phys. Rev. D 41, 403 (1990).

[21] H. Otsuki and T. Futamase, Prog. Theor. Phys. 85, 721 (1991).
[22] S.W. Hawking, Comm. Math. Phys. 25, 167 (1972).

[23] R. Ruffini and J.A. Wheeler, Phys. Today 24 (1), 30 (1971); J. Chase, Comm. Math. Phys. 19, 276 (1970); J.D. Bekenstein, Phys. Rev. D 5, 1239 (1972); Phys. Rev. D 5, 2403 (1972); Phys. Rev. Lett. 28, 452 (1972); arXiv:gr-qc/9605059 C. Teitelboim, Lett. Nuovo Cimento 3S2, 326 (1972); T. Zannias, J. Math. Phys. 36, 6970 (1995); J.D. Bekenstein, Phys. Rev. D 51, 6608 (1995); A. Saa, J. Math. Phys. 37, 2346 (1996); K.A. Bronnikov, Phys. Rev. D 64, 064013 (2001). C.A.R. Herdeiro and E. Radu, Int. J. Mod. Phys. D 24, 1542014 (2015); T.P. Sotiriou, Class. Quantum Grav. 32, 214002 (2015).

[24] T.P. Sotiriou and V. Faraoni, Phys. Rev. Lett. 108, 081103 (2012).

[25] S. Bhattacharya, K.F. Dialektopoulos, A.E. Romano, and T.N. Tomaras, Phys. Rev. Lett. 115, 181104 (2015).

[26] K. Henttunen, T. Multamäki, and I. Viljia, Phys. Rev. D 77, 024040 (2008); K. Henttunen and I. Viljia, Phys. Lett. $B$ 731, 110 (2014); K. Henttunen and I. Viljia, J. Cosmol. Astropart. Phys. 1505, 001 (2015).

[27] S. Capozziello and M. De Laurentis, Nuovo Cimento 38 C, 156 (2015).

[28] R.M. Wald, General Relativity (Chicago University Press, Chicago, 1984).

[29] J. Podolský and O. Hruška, Phys. Rev. D 95, 124052 (2017).

[30] A.H. Abbassi, J. High Energy Phys. 04, 011 (1999); A.H. Abbassi, S. Gharanfoli, and A.M. Abbassi, Apeiron 9, 1 (2002).

[31] K.A. Meissner, arXiv:0901.0640.

[32] G.C. McVittie, Mon. Not. Roy. Astron. Soc. 93, 325 (1933).

[33] V. Faraoni and A. Jacques, Phys. Rev. D 76, 063510 (2007).

[34] B.C. Nolan, Class. Quantum Grav. 16, 1227 (1999); G.W. Gibbons, C.M. Warnick, and M.C. Werner, Class. Quantum Grav. 25, 245009 (2008); N. Kaloper, M. Kleban, and D. Martin, Phys. Rev. D 81, 104044 (2010); J.P. Mimoso, M. Le Delliou, and F.C. Mena, Phys. Rev. D 81, 123514 (2010); V. Faraoni, A.F. Zambrano Moreno, and R. Nandra, Phys. Rev. D 85, 08352 (2012); V. Faraoni and A.F. Zambrano Moreno, Phys. Rev. D 88, 044011 (2012); M. Le Delliou, F.C. Mena, and J.P. Mimoso, Phys. Rev. D 83, 103528 (2011); K. Lake and M. Abdelqader, Phys. Rev. D 84, 044045 (2011); D.C. Guariento, M. Fontanini, A.M. da Silva, and E. Abdalla, Phys. Rev. D 86, 124020 (2012); M. Le Delliou, J.P. Mimoso, 
F.C. Mena, M. Fontanini, D.C. Guariento, and E. Abdalla, Phys. Rev. D 88, 027301 (2013); A.M. da Silva, M. Fontanini, and D.C. Guariento, Phys. Rev. D 87, 064030 (2013); J.P. Mimoso, M. Le Delliou, and F.C. Mena, Phys. Rev. D 88, 043501 (2013); V. Faraoni, A.F. Zambrano Moreno, and A. Prain, Phys. Rev. D 89, 103514 (2014); A. Maciel, M. Le Delliou, and J.P. Mimoso, Phys. Rev. D 92, 083525 (2015); A. Maciel, D.C. Guariento, and C. Molina, Phys. Rev. D 91, 084043 (2015); O.F. Piattella, Phys. Rev. D 93, 024020 (2016); M.E. Aghili, B. Bolen, and L. Bombelli, Gen. Relat. Gravit. 49, 10 (2017); V. Faraoni and M. Lapierre-Léonard, Phys. Rev. D 95, 023509 (2017).

[35] R. Nandra, A.N. Lasenby, and M.P. Hobson, Mon. Not. Roy. Astron. Soc. 422, 2931 (2012); Mon. Not. Roy. Astron. Soc. 422, 2945 (2012).

[36] V. Faraoni, Cosmological and Black Hole Apparent Horizons (Springer, New York, 2015).

[37] H. Arakida, Gen. Relat. Gravit. 43, 2127 (2011).

[38] C. Gao, X. Chen, V. Faraoni, and Y.-G. Shen, Phys. Rev. D 78, 024008 (2008).

[39] N. Afshordi, D.J.H. Chung, M. Doran, G. Geshnizjani, Phys. Rev. D 75, 123509 (2007); N. Afshordi, Phys. Rev. D 80, 081502 (2009).

[40] N. Afshordi, M. Fontanini, and D.C. Guariento, Phys.
Rev. D 90, 084012 (2014); E. Abdalla, N. Afshordi, M. Fontanini, D.C. Guariento, and E. Papantonopoulos, Phys. Rev. D 89, 104018 (2014).

[41] S.N.G. Thakurta, Indian J. Phys. 55B, 304 (1981).

[42] M.M.C. Mello, A. Maciel, and V. Zanchin, Phys. Rev. D 95, 084031 (2017).

[43] H. Culetu, J. Phys. Conf. Ser. 437, 012005 (2013).

[44] D.D. McNutt and D.N. Page, Phys. Rev. D 95, 084044 (2017).

[45] V. Faraoni, Phys. Rev. D 80, 044013 (2009).

[46] V. Faraoni, C. Gao, X. Chen, and Y.-G. Shen, Phys. Lett. $B$ 671, 7 (2009).

[47] T. Clifton, D.F. Mota, and J.D. Barrow, Mon. Not. Roy. Astron. Soc. 358, 601 (2005).

[48] P. Castelo Ferreira, Phys. Lett. B 684, 73 (2010) (2010); Adv. Sp. Res. 51, 1266 (2013); arXiv:0907.0847 arXiv:1203.1844

[49] R.M. Wald, Phys. Rev. D 28, 2118 (1983); M.S. Turner and L.M. Widrow, Phys. Rev. Lett. 57, 2237 (1986); L. Jensen and J.A. Stein-Schabes, Phys. Rev. D 35, 1146 (1987).

[50] V. Faraoni and W. Israel, Phys. Rev. D 71, 064017 (2005).

[51] R.R. Caldwell, M. Kamionkowski, and N.N. Weinberg, Phys. Rev. Lett. 91, 071301 (2003); S. Nesseris and L. Perivolaropoulos, Phys. Rev. D 70, 123529 (2004). 